\title{
Revisiting of Channel Access Mechanisms in Mobile Wireless Networks through Exploiting Physical Layer Technologies
}

\author{
Junmei Yao, ${ }^{1}$ Jun Xu, ${ }^{1}$ Yue Ling Che, ${ }^{1}$ Kaishun Wu $\mathbb{D},{ }^{1}$ and Wei Lou $\mathbb{D}^{2}$ \\ ${ }^{1}$ College of Computer Science and Software Engineering, Shenzhen University, Shenzhen, China \\ ${ }^{2}$ Department of Computing, The Hong Kong Polytechnic University, Kowloon, Hong Kong \\ Correspondence should be addressed to Kaishun Wu; wu@szu.edu.cn
}

Received 16 January 2018; Accepted 4 April 2018; Published 15 May 2018

Academic Editor: Ziming Zhao

Copyright (C) 2018 Junmei Yao et al. This is an open access article distributed under the Creative Commons Attribution License, which permits unrestricted use, distribution, and reproduction in any medium, provided the original work is properly cited.

\begin{abstract}
The wireless local area networks (WLANs) have been widely deployed with the rapid development of mobile devices and have further been brought into new applications with infrastructure mobility due to the growth of unmanned aerial vehicles (UAVs). However, the WLANs still face persistent challenge on increasing the network throughput to meet the customer's requirement and fight against the node mobility. Interference is a well-known issue that would degrade the network performance due to the broadcast characteristics of the wireless signals. Moreover, with infrastructure mobility, the interference becomes the key obstacle in pursuing the channel capacity. Legacy interference management mechanism through the channel access control in the MAC layer design of the 802.11 standard has some well-known drawbacks, such as exposed and hidden terminal problems, inefficient rate adaptation, and retransmission schemes, making the efficient interference management an everlasting research topic over the years. Recently, interference management through exploiting physical layer mechanisms has attracted much research interest and has been proven to be a promising way to improve the network throughput, especially under the infrastructure mobility scenarios which provides more indicators for node dynamics. In this paper, we introduce a series of representative physical layer techniques and analyze how they are exploited for interference management to improve the network performance. We also provide some discussions about the research challenges and give potential future research topics in this area.
\end{abstract}

\section{Introduction}

With the rapid development of mobile devices, the wireless local area networks (WLANs, often called Wi-Fi networks) have been widely deployed in various places, from home to office, restaurants, clubs, etc., due to its acceptable performance and easy-to-deploy characteristics [1]. Currently, the growth of unmanned aerial vehicles (UAVs) brings these networks into new applications with infrastructure mobility, where the access point (AP) is mobile to alleviate some unpredictable problems, such as natural disaster and poor coverage. However, the WLANs still face a continual challenge on the channel capacity owing to the huge amount of mobile data traffic. As forecast by the Cisco Visual Networking Index [2] (VNI), the global mobile data traffic will increase sevenfold from 2016 to 2021, and about 50\% of the traffic will be offloaded to WLANs.

Actually, the IEEE task group has made great efforts on throughput improvement in WLANs through the evolution of the 802.11 standards in the past twenty years. From 1997 when the base version of IEEE 802.11 standard which supports $1 / 2 \mathrm{Mbps}$ data rate was released, a series of 802.11 standards have been released to increase the physical layer data rate. $802.11 \mathrm{~b}$ and $802.11 \mathrm{a} / \mathrm{g}$ increase the data rate of single stream up to $11 \mathrm{Mbps}$ and $54 \mathrm{Mbps}$, respectively, through exploiting higher-order modulations; 802.11n and 802.11ac increase the data transmission rate up to $600 \mathrm{Mbps}$ and $>6 \mathrm{Gbps}$, respectively, through further exploiting the Multiple Input and Multiple Output (MIMO) technique. Now an upcoming 802.11ax aims to achieve up to $10 \mathrm{Gbps}$ data rate 
and is expected to be released in 2019. However, there is a huge gap between the physical layer data rate and the network throughput [3], which is mainly due to the inefficient channel access mechanism for interference management in wireless mobile networks.

Interference is well known to degrade the network throughput due to the broadcast characteristics of the wireless signals. Theoretically, the interference occurs when the received signal's Signal to Interference and Noise Ratio (SINR) is below a required threshold. The problem will be even worse under the infrastructure mobility scenario as the mobile APs may lead to the dynamic change of the received signal power. The 802.11 family recommends the carrier sense multiple access (CSMA) mechanism in the MAC layer to manage interference. A transmitter can proceed its transmission only when it determines that the channel is idle; otherwise, it should keep silent until the channel becomes idle to avoid interference. This mechanism is simple but inefficient, as it has the following well-known drawbacks which may result in low network performance: (1) it may fail to avoid interference effectively due to the hidden terminal problem, where a node will interfere with an ongoing link as it cannot sense the data transmission from the transmitter but can interfere with the data reception at the receiver; (2) it may prohibit concurrent transmissions of noninterfering links due to the exposed terminal problem, where a node is prohibited to transmit signals as it can hear the data transmission from the transmitter, although it will not interfere with the data reception at the receiver; (3) it may be unable to transmit packets in the optimal data rate according to the actual channel situations, while data can certainly be transmitted at a higher rate when the wireless channel is better; (4) it has an inefficient retransmission scheme when a data packet is not detected correctly, as it makes the whole data packet retransmitted although a large partial of this packet can be detected correctly. The above listed drawbacks would degrade the network performance to a great extent in some situations and thus motivate the researchers to work on more effective interference management mechanisms.

Exploiting physical layer techniques is a promising way to manage interference from one or more of the above four aspects and has attracted much research interest in the recent decade. The benefits brought by the physical layer techniques, such as interference resistance and real-time channel estimation, inspire a new way for designing high efficient channel access mechanisms, especially under the infrastructure mobility situations. This paper will present a series of physical layer techniques and investigate how they are exploited for interference management, as shown in Figure 1. Cross correlation has the high interference-resistant characteristic and can tolerate the collision of control or data packets to some extent; it is always utilized to combat the exposed and hidden terminal problems or reduce the coordination overhead. SoftPHY provides each received bit's physical layer confidence to the upper layer [4], so as to determine which bits need to be retransmitted and what the optimal data rate would be. Successive interference cancellation (SIC) makes a strong interfered signal detected at first to recover an inferior strong signal, if the SINR is above the threshold after subtracting the strongest one [5]; it is mainly utilized to increase concurrent transmissions. Rateless coding aims to make the signal with fixed coding and modulation schemes decoded at any SNR environments, through making the signal transmitted multiple times and combining them at the physical layer of the receiver effectively, thus achieving an optimal data rate [6]. Some physical layer unique features are also exploited to design high efficient interference management mechanism; for example, the redundancy design for communication is commonly utilized to convey the coordination information, assisting in avoiding interference, increasing concurrent transmissions, or reducing coordination overhead.

Although with demonstrated high performance through hardware experiments or simulations, the adoption of these techniques represents some major challenges in current wireless networks, especially the high computational overhead in signal process, inflexible MAC layer design due to the scenario limitation of the techniques, etc. This survey will also investigate the challenges of each physical layer technique when applying to real networks for interference management.

The rest of this paper is organized as follows: Section 2 gives an overview of the IEEE 802.11 family, including its physical layer and MAC layer specifications. Section 3 discusses the problems in the legacy channel access mechanism recommended by the IEEE 802.11 MAC. Section 4 surveys a series of physical layer techniques and how they would be applied for interference management. Section 5 discusses advantages and limitations of each kind of interference management mechanisms and also puts forwards some future directions in this research area. Section 6 concludes this paper.

\section{Review of IEEE 802.11 Standard}

This section will briefly introduce the standards in the IEEE 802.11 family, including the physical layer (PHY) and MAC specifications.

2.1. The IEEE 802.11 PHY Specification. In the past twenty years, the IEEE 802.11 task group has made great efforts to increase the data rate of wireless devices through the physical layer evolutions.

As shown in Table 1, the 802.11 base version was released in 1997, which specifies two data rates of $1 / 2 \mathrm{Mbps}$ working in the $2.4 \mathrm{GHz}$ frequency band and uses the Direct-Sequence Spread Spectrum (DSSS) and Frequency Hopping Spread Spectrum (FHSS) as the modulation types. After that, they released a series of 802.11 standards to increase the physical layer data rate. In 1999, 802.11a, which operates in the $5 \mathrm{GHz}$ frequency band and uses the Orthogonal Frequency Division Multiplexing (OFDM) as the modulation type, was introduced to enhance the data rate to $54 \mathrm{Mbps}$. In the same year, 802.11b, which operates in the same frequency band and uses the same modulation technique with the base version, was also introduced to support up to $11 \mathrm{Mbps}$ data rate. Due to its dramatic throughput increase and the similar PHY technique compared with the base version, the 802.11b becomes the definitive WLAN technology. In 2003, 
TABLE 1: IEEE 802.11 standards for throughput improvement.

\begin{tabular}{|c|c|c|c|c|c|}
\hline Version & Release Time & $\begin{array}{c}\text { Maximum Data } \\
\text { Rate }\end{array}$ & $\begin{array}{c}\text { Frequency } \\
\text { Band }(\mathrm{GHz})\end{array}$ & $\begin{array}{l}\text { Bandwidth } \\
(\mathrm{MHz})\end{array}$ & Modulation \\
\hline $\begin{array}{l}\text { 802.11-base } \\
\text { version }\end{array}$ & Jun. 1997 & 2 Mbps & 2.4 & 20 & $\begin{array}{c}\text { BPSK, QPSK } \\
\text { DSSS, FHSS }\end{array}$ \\
\hline $802.11 \mathrm{~b}$ & Sep. 1999 & $11 \mathrm{Mbps}$ & 2.4 & 20 & $\begin{array}{l}\text { BPSK, QPSK } \\
\text { DSSS (CCK) } \\
\end{array}$ \\
\hline $802.11 \mathrm{a}$ & Sep. 1999 & $54 \mathrm{Mbps}$ & 5 & 20 & $\begin{array}{c}\text { BPSK, QPSK, 16-QAM, } \\
\text { 64-QAM } \\
\text { OFDM } \\
\end{array}$ \\
\hline 802.11g & Jun. 2003 & $54 \mathrm{Mbps}$ & 2.4 & 20 & $\begin{array}{c}\text { BPSK, QPSK, 16-QAM, } \\
\text { 64-QAM } \\
\text { OFDM, DSSS }\end{array}$ \\
\hline $802.11 \mathrm{n}$ & Oct. 2009 & $600 \mathrm{Mbps}$ & $2.4 \& 5$ & 20,40 & $\begin{array}{c}\text { BPSK, QPSK, 16-QAM, } \\
\text { 64-QAM } \\
\text { OFDM, MIMO }\end{array}$ \\
\hline $802.11 \mathrm{ac}$ & Dec. 2013 & $6.933 \mathrm{Gbps}$ & $2.4 \& 5$ & $20,40,80,160$ & $\begin{array}{c}\text { BPSK, QPSK, } \\
\text { 16-QAM, 64-QAM, } \\
\text { 256-QAM } \\
\text { OFDM, MIMO, } \\
\text { MU-MIMO }\end{array}$ \\
\hline 802.11ax & Approx. 2019 & $>10$ Gbps & $<6$ & ---- & $\begin{array}{c}\text { BPSK, QPSK, 16-QAM, } \\
\text { 64-QAM, } \\
\text { 256-QAM, 1024-QAM, } \\
\text { OFDM, MIMO, } \\
\text { MU-MIMO, OFDMA }\end{array}$ \\
\hline
\end{tabular}

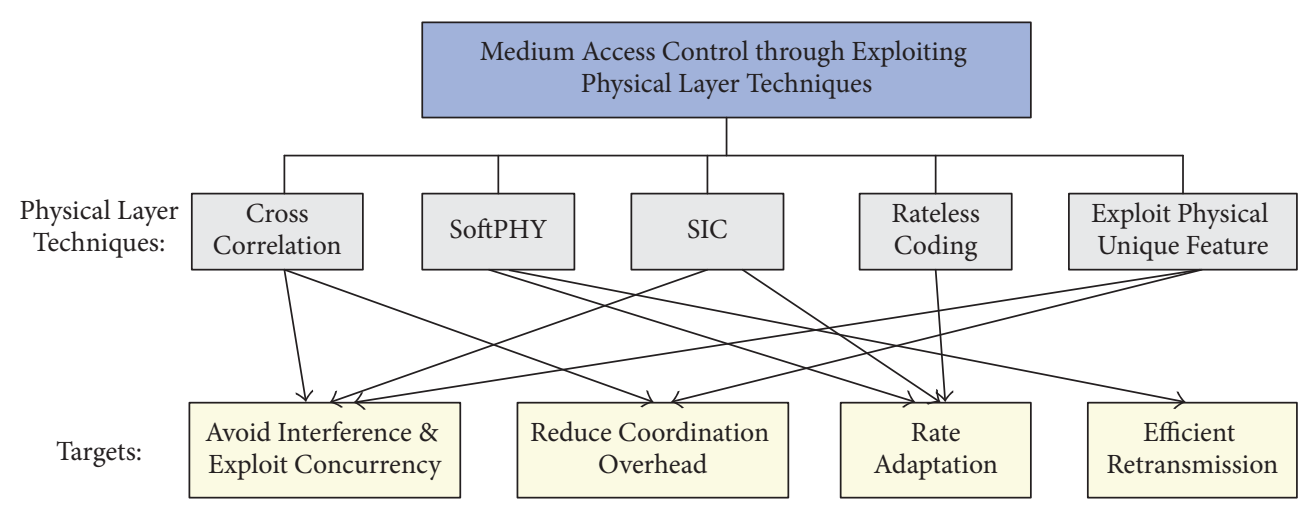

FIGURE 1: The classification of interference management mechanisms through exploiting physical layer techniques in WLANs.

the IEEE task group released 802.11g, which uses OFDM as its modulation technique (the same as 802.11a), and finally achieves up to $54 \mathrm{Mbps}$ data rate in the $2.4 \mathrm{GHz}$ band. It is always regarded as an extension of $802.11 \mathrm{~b}$.

The utilizing of Multiple Input and Multiple Output (MIMO) antennas substantially improves the spectral efficiency and finally improves the physical data rate of WLANs. In 2009, 802.11n, the first standard which supports MIMO, was ratified to support up to $600 \mathrm{Mbps}$ data rate through four spatial streams and $40 \mathrm{MHz}$ wider bandwidth. The latest released version is $802.11 \mathrm{ac}$, which increases the data rate to >6 Gbps through $160 \mathrm{MHz}$ wider bandwidth, eight spatial streams, higher-order 256-QAM modulation, and MultiUser MIMO (MU-MIMO). The upcoming 802.11ax, which is currently in an early stage of development, is predicted to achieve $>10$ Gbps data rate, through further introducing the Orthogonal Frequency Division Multiple Access (OFDMA) technique and the higher-order 1024-QAM modulation.

To illustrate the evolution of the physical layer process in WLANs more clearly, we show its basic diagram at the transmitter side, as shown in Figure 2. The receiver has the reverse processes. The components with dashed lines, including spreader, OFDM modulator, and MIMO encoder, are optional for specific 802.11 standard which supports the corresponding functions.

2.2. The IEEE 802.11 MAC Specification. Although the physical layer techniques have been updated to higher-order modulations, wider bandwidth and MIMO to increase the data rate, the interference management process which is 


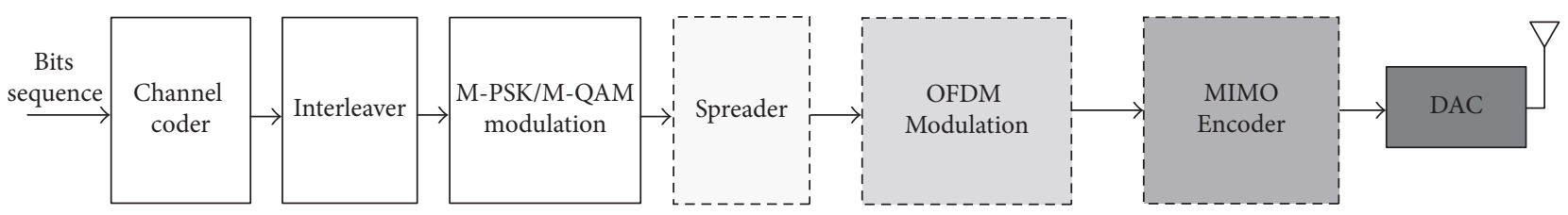

FIgURE 2: The basic diagram of the physical layer process at the transmitter in WLANs. The receiver has the reverse process.

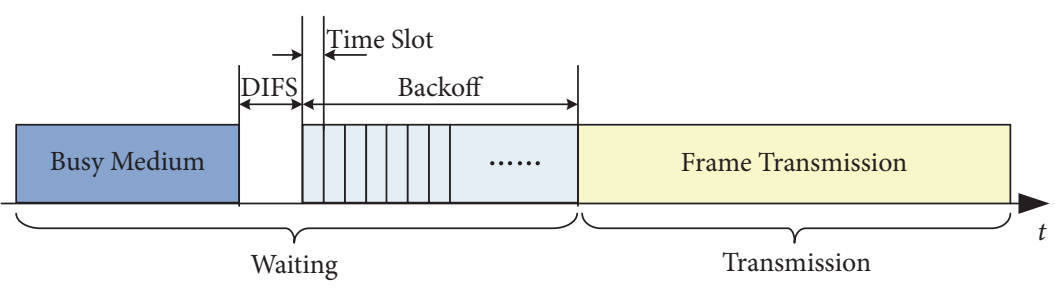

FIGURE 3: The basic access mechanism in DCF.

defined in the MAC layer of the 802.11 base version has only a little change along with the update of the standards. The 802.11 MAC recommends two kinds of coordination functions for interference management: a distributed mechanism called Distributed Coordination Function (DCF) and a centralized mechanism called Point Coordination Function (PCF). DCF is widely deployed in current WLANs, while PCF is not implemented in most devices as it is not part of the Wi-Fi Alliance's standard.

DCF further contains two mechanisms: a physical carrier sense mechanism called carrier sense multiple access (CSMA) and a virtual carrier sense mechanism called RTS/CTS. Both of them are designed to reduce the collision probability in a distributed way when multiple nodes access the same channel.

The CSMA and RTS/CTS follow the same basic channel access mechanism, as shown in Figure 3. Before transmitting, a sender first senses the medium to determine whether the channel is available; if the channel is determined to be busy when a nearby node is transmitting a signal, the sender should defer its transmission until the channel is available again to avoid interfering with the ongoing link. When the channel is idle for a DIFS (distributed interframe space) period, the node shall generate a random backoff period for an additional deferral time before transmitting. The backoff period is decremented along with the time when the channel stays idle and is suspended otherwise. Only when the backoff period expires can the node transmit its signal.

When the backoff expires, the two channel access mechanisms have different operations. CSMA makes the node transmit its data packet immediately, while RTS/CTS utilizes the exchange of the RTS and CTS frames to reserve medium for the following data transmission, through the NAV value carried in both frames; all the neighboring nodes that receive the RTS or CTS frame will keep silent during the NAV time to avoid interfering with this data transmission. For both mechanisms, the receiver should reply an acknowledgment (ACK) after receiving the data packet; the transmitter determines the data transmission to be successful only if it detects the ACK from the receiver; otherwise, it will retransmit this packet.

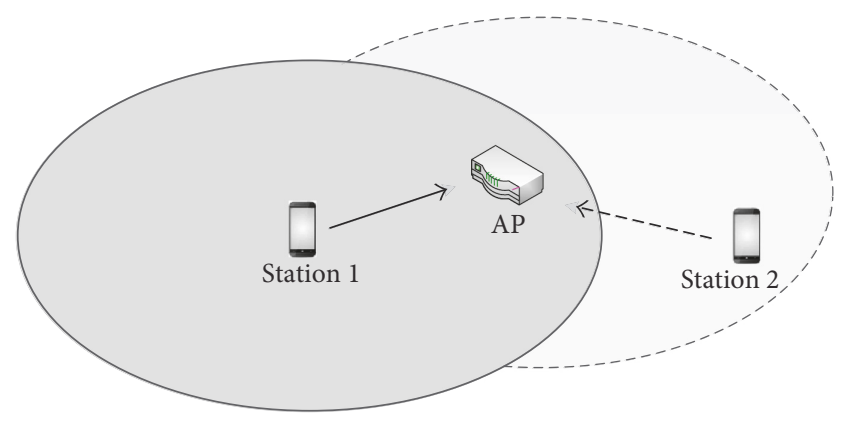

Coverage area of Station 1

Coverage area of AP

Figure 4: A scenario of the hidden terminal problem.

\section{Problems in Legacy Channel Access Mechanism}

In this section, we will give detailed description of four problems in current channel access mechanism.

3.1. The Hidden and Exposed Terminal Problems. CSMA has a serious hidden terminal problem which induces collisions, as it uses the channel situation at the transmitter side to determine that at the receiver side, which process is always improper in the real networks. As shown in Figure 4, when Station 1 is transmitting a data packet to the AP, Station 2 may determine that the channel is idle as it is far from Station 1 and out of its coverage area, it shall transmit signal simultaneously. This transmission will interfere with the AP's data reception from Station 1, making it not detect the data packet correctly. RTS/CTS is proposed to mainly solve this problem through the CTS frame transmitted by the receiver. According to the RTS/CTS mechanism, as Station 2 can receive the CTS frame transmitted by AP, it will keep silence during this transmission, thus avoiding inducing collisions.

However, both CSMA and RTS/CTS have the exposed terminal problem which prohibits concurrent transmissions. 


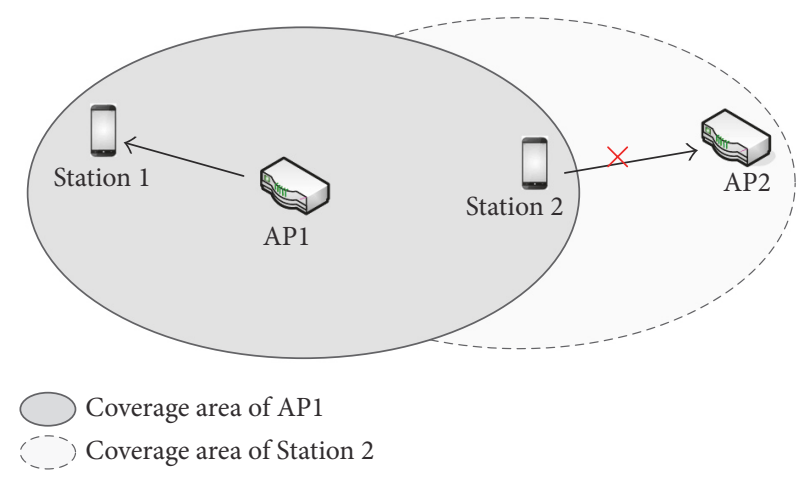

FIGURE 5: A scenario of the exposed terminal problem.

As shown in Figure 5, when AP1 is transmitting a data packet to Station 1, Station 2 which is near to AP1 is prohibited to transmit signals to AP2 simultaneously as it determines the channel is busy, although the data packet transmissions of these two links have no mutual interference.

3.2. The Coordination Overhead. There are two main kinds of coordination overhead in the 802.11 standard that may degrade the network throughput significantly: the backoff and the control frames' transmission overhead.

As shown in Figure 3, the backoff time will obviously induce a large amount of overhead as the nodes should wait for a long time before transmission, especially in the case when multiple nodes conduct the backoff simultaneously. In addition, a failure data transmission will lead to exponential increase in the backoff period and thus further increase the overhead. Researchers have pointed out that backoff leads to more than $30 \%$ throughput degradation in the 802.11 bystem [7], and the results would be even worse in the higher data rate situation [8].

The RTS, CTS, and ACK control frames' transmissions also induce too much overhead to the system, especially in the situation of the high data packet transmission rate, as the RTS and CTS are still transmitted at the lowest rate. For this reason, the current widely used mechanism is CSMA but not the RTS/CTS, although RTS/CTS can solve the hidden terminal problem efficiently.

3.3. Rate Adaptation Problem. The 802.11 standard recommends a set of data transmission rates which are related to different modulations, coding types, and number of spatial streams for MIMO. Table 2 lists the eight data rates supported by $802.11 \mathrm{a}$ as an example. Due to the different required SNR threshold for each data rate, it is easy to understand that optimizing the data transmission rate according to the channel quality can significantly improve the network throughput. The 802.11 standard does not give any recommendation for the rate adaptation process, leaving it an open research topic till now.

3.4. Low Efficient Retransmission. According to the DCF process, the transmitter determines the data transmission to be successful only if it detects the ACK from the receiver;
TABLE 2: Data rates supported by $802.11 \mathrm{~A}$.

\begin{tabular}{lccc}
\hline $\begin{array}{l}\text { Data rate } \\
(\mathrm{Mbps})\end{array}$ & $\begin{array}{c}\text { SNR threshold } \\
(\mathrm{dB})\end{array}$ & $\begin{array}{c}\text { Modulation } \\
\text { scheme }\end{array}$ & Coding rate \\
\hline 6 & 6.02 & BPSK & $1 / 2$ \\
9 & 7.78 & BPSK & $3 / 4$ \\
12 & 9.03 & QPSK & $1 / 2$ \\
18 & 10.79 & QPSK & $3 / 4$ \\
24 & 17.04 & 16-QAM & $1 / 2$ \\
36 & 18.80 & 16-QAM & $3 / 4$ \\
48 & 24.05 & 64-QAM & $1 / 2$ \\
54 & 24.56 & 64-QAM & $3 / 4$ \\
\hline
\end{tabular}

otherwise, it will retransmit the whole data packet. However, as observed by researchers that the wireless channel often induces errors to only a few bits of a packet [4], retransmission of the entire packet is wasteful as the correct bits are transmitted for multiple times. How to organize the incorrect bits and make them retransmitted effectively also attracts much research interest.

3.5. Discussion. There has already been a great amount of research on solving one or more of these problems to improve the network throughput through upper layer design, such as constructing an interference map to solve the exposed and hidden terminal problems [9], scheduling the data transmissions centrally to reduce the coordination overhead [10], adapting the transmission rate through observing either the packet loss rate or the SNR at the receiver side [11], etc. However, the mechanisms through exploiting physical layer techniques become more promising in recent years, which will be described in the following part.

\section{Survey on Interference Management through Exploiting Physical Layer Techniques}

In this section, we will investigate a series of physical layer techniques and how they are explored for interference management in wireless networks, as shown in Figure 1. 

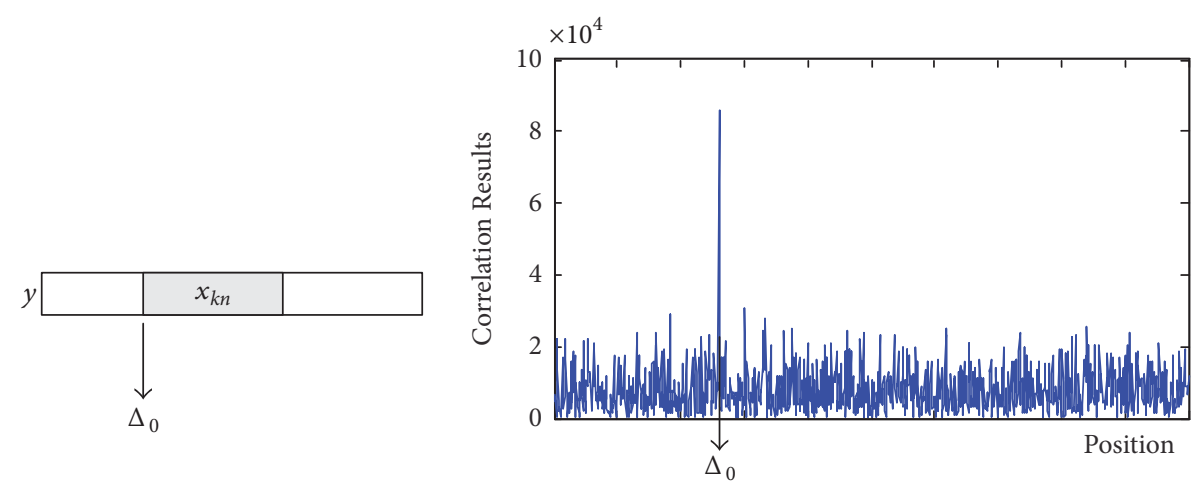

FIGURE 6: When a known sequence $x_{k n}$ is in the received signal $y$ at the position $\Delta_{0}$, the correlation results between $y$ and $x_{k n}$ will have a peak value at $\Delta_{0}$, while those at other positions will have small values.

4.1. Cross Correlation. The technology of cross correlation has been proposed for many years, and it has already been used in WLANs in the preamble synchronization and the DSSS modulation process when the 802.11 standard was established in 1997. However, it begins to be utilized for the purpose of interference management in WLANs only from about 2008 .

4.1.1. Introduction of Cross Correlation. In the communication systems, cross correlation is commonly used to search for a known transmitted sequence in the received signal. According to the digital communication, a wireless signal is represented as a stream of complex samples, and a received signal $y[n]$ is different from the transmitted one $x[n]$ in amplitude, frequency, and phase, due to the channel attenuation, multiple path, the difference in the oscillator, and so on. Their relationship is always represented as $y[n]=H$. $x[n] e^{j 2 \pi \delta_{f} T+\theta_{0}}$, where $H$ is the amplitude attenuation factor, $\delta_{f}$ is the frequency offset, $T$ is the sampling period, and $\theta_{0}$ is the initial phase offset. When a node receives a signal $y[n]$ and intends to figure out whether a known sequence $x_{k n}[i](i=$ $\{1, \ldots, L\})$ is received, it will calculate the correlation results between $y$ and $x_{k n}$ at each position of $y$, through the equation $R[\Delta]=\sum_{k=1}^{L} \overline{x_{k n}[k]} \cdot y[\Delta+k]$. As an example shown in Figure 6, the value of $R[\Delta]$ will be very small except that at the position $\Delta_{0}$, where $x_{k n}$ begins to appear.

4.1.2. Survey on Cross Correlation. This technology has been utilized to both combat the exposed and hidden terminals and reduce the coordination overhead.

(i) Avoid Interference and Exploit Concurrency. Many current strategies leverage the technology of cross correlation to recover the control information under interferences so as to coordinate between nodes [12-17]. In these protocols, authors carefully design some known symbol sequences to convey the control information and conduct the cross correlation between the received signal and the known sequences to determine which sequence is received, so as to obtain the control information under interferences, as shown in Figure 7.

CSMA/CN [13] attempts to implement the similar CSMA/CD (carrier sense multiple access/collision detection,

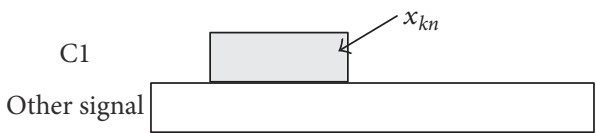

FIgURE 7: A scenario of permitting the control frame $\mathrm{Cl}$ collided by other signals. The known sequence $x_{k n}$ here is $\mathrm{C} 1$ which can be detected correctly through cross correlation. Researchers exploit this method to reduce the control frames' transmission overhead, increase concurrent transmissions, and avoid interference more efficiently.

a well-known collision avoidance mechanism in the wired networks) to wireless networks. During a data packet reception, it makes the receiver transmit a collision notification information immediately when a collision occurs, through utilizing the correlated symbol sequences. The transmitter can then detect this sequence correctly under strong interference and abort the transmission instantly to avoid further collisions. 802.11ec [15] exploits the cross correlation to accomplish the control frames' transmissions. Comparing with the 802.11 standard, this protocol uses three kinds of known sequences to convey the RTS, CTS, and ACK information. As the known sequences can tolerate strong interferences, and the duration of these sequences is much less than that of the corresponding packets, this protocol can improve the network throughput through both avoiding collisions and reducing the transmission overhead of the control frames. RTS/S-CTS [14] presents a symbol-level detection mechanism to combat both the CTS collision problem and the remote hidden terminal problem, as the symbol sequences that carry useful information in the new S-CTS packet can be detected in very low SINR and SNR environments. IRMA [16] proposes to solve the exposed terminal problem through exploiting this technology. It designs a physical layer mechanism, called signature detection, to combat the CTS/ACK collisions at the transmitter side. It also designs new mechanisms in the MAC layer to differentiate the interfering and noninterfering links. Both mechanisms collaborate to exploit concurrent transmissions and avoid interference.

Some other researchers exploit this technology to recover the collided data signal. The first work is Zigzag [18], as shown in Figure 8. It focuses on the uplink transmissions from 


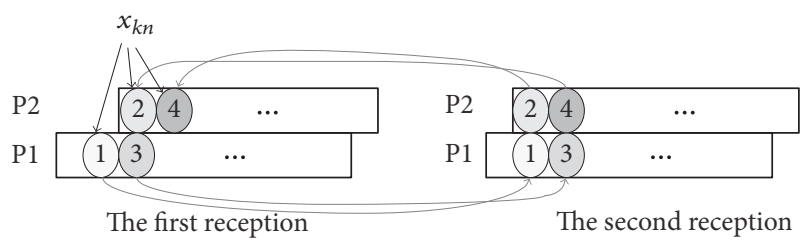

FIGURE 8: A scenario of detecting two collided data packets P1 and P2. This is a classical scenario of Zigzag, which lets the collided signals be transmitted again. The known sequence $x_{k n}$ here is the collision-free or detected chunks. Zigzag first decodes the collisionfree chunk 1 of $\mathrm{P} 1$ in the first reception, subtracts it in the second reception to decode chunk 2 of $\mathrm{P} 2$, then subtracts chunk 2 to decode chunk 3 in P1, etc. Through this way, it iteratively decodes both packets, thus solving the hidden terminal problems shown in Figure 4.

clients to AP and lets the collided signals be transmitted again for decoding. As these collisions have different interferencefree blocks, it then exploits this collision diversity to bootstrap its decoding. Figure 8 can be extended to $n$-collided packets scenario. This method can solve the hidden terminal problem and achieve the same throughput as if the colliding packets were scheduled in a TDMA way. Symphony [19] extends the idea of Zigzag in the multiple-AP scenarios through utilizing the wired backbone among APs. It encourages collisions of packets transmitted from clients to APs and cooperatively decodes all the packets through the Zigzag-like process. This kind of idea has also been extended to some other scenarios, such as the wireless cooperative relay in DAC [20] and efficient broadcasting in Chorus [21].

(ii) Reduce Coordination Overhead. Researchers also exploit this technology to reduce the coordination overhead in backoff. The semidistributed backoff (SDB) algorithm in [22] is proposed to make nodes perform the receiver side backoff. Using SDB algorithm, they design a MAC protocol SemiDCF, which exploits the collision detection capability of receivers for disseminating information on optimal backoffs to the contenders using signature vectors, so as to migrate backoff from random to deterministic and largely reduce the backoff time in 802.11 standard. CWM [23] exploits collision tolerance mechanism to reduce the backoff time and improve the channel utilization in the wireless networks. Upon detecting a collided signal from multiple senders, the receiver obtains the senders' IDs through exploiting the correlatable preamble in the physical layer and then allocates each sender a different timeslot so that the senders can transmit their data packets one after another in the following time, without mutual interference.

\subsection{SoftPHY}

4.2.1. Introduction of SoftPHY. The SoftPHY was first introduced in [24] to provide PHY-independent hints about the PHY's confidence in each bit to the upper layer, so as to determine which bits need to be retransmitted. As shown in Figure 9(a), the basic idea of SoftPHY is to calculate the distance $\varphi$ between the received signal's constellation point $y_{i}$ and the corresponding theoretical point $a_{i}$; that is, $\varphi_{i}=K_{c} \cdot\left\|y_{i}-a_{i}\right\|$, where $K_{c}$ is a constant factor related to the modulation type.

The SoftPHY is designed to be suitable with the real communication systems, where redundancy is added through coding to make the data transmission more robust to the interference existing in the wireless channel. That means the node maps each $b$-bit string to a $B$-bit codeword $C_{i}(b<B)$, while the codeword is selected from a codebook $\left\{C_{i}, \ldots, C_{M}\right\}$. The $B$-bit codeword will be transformed to $B / k$ symbols after modulation process, where $k$ indicates the number of bits per symbol and is decided by the modulation type. Then the SoftPHY hint for any $B$-bit codeword is $\varphi=\sum_{i=1}^{B / k} \varphi_{i}$. The codeword with a larger $\varphi$ value is determined to be detected correctly with higher probability and higher confidence.

4.2.2. Survey on SoftPHY. This technology has been mainly utilized either to increase the retransmission efficiency or for rate adaptation.

(i) Efficient Retransmission. Through observing that current wireless protocols make all the data packet retransmitted even when detecting a small number of bit errors, authors in [24] propose partial packet recovery (PPR) based on the SoftPHY interface to improve the retransmission efficiency. PPR contains a link-layer protocol design which permits a receiver to encode a retransmission request, so that the transmitter will only retransmit the bits with low confidence. It also contains a postamble scheme which can be utilized to recover the packet in a "roll back" way when the preamble is collided, so as to further improve the network throughput.

(ii) Rate Adaptation. Different from PPR [24] where SoftPHY is utilized to estimate the confidence of symbols for retransmission determination, SoftRate [25] begins to exploit the SoftPHY hints to estimate the BER of a received frame for rate adaptation. Nodes use this BER estimation to pick up optimal bit rates for the next frame transmission and thus achieve the rate adaptation on the frame level. This mechanism can rapidly respond to the varied channel conditions. It can also identify whether the changes in the BER estimation are induced by interference and only apply rate adaptation in the situation of channel errors. However, authors in [26] point out that it is very hard for one node to directly jump to the best rate according to the BER estimation. They propose AccuRate to "replay" the signal dispersion in the channel on all possible rates and select the data rate which achieves optimal throughput. This "replay" action is simulated at the receiver and there is no need for the transmitter to transmit signals each time. AccuRate can outperform SoftRate at the expense of implementation complexity.

Authors in [27] utilize Log-Likelihood Ratio (LLR) estimation to determine the confidence of each demodulated bits and make those with low confidence retransmitted. Meanwhile, they also leverage these low-confidence bits and combine them from multiple failed transmissions by adding up their LLR. All the results will be fed into the FEC decoder to increase the decoding efficiency. Due to the lower retransmission overhead in this mechanism, a higher data rate can be selected to improve the channel capacity. 


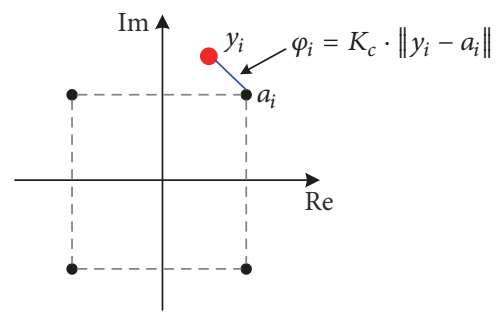

(a) SoftPHY hint calculation for each symbol in uncoded channel

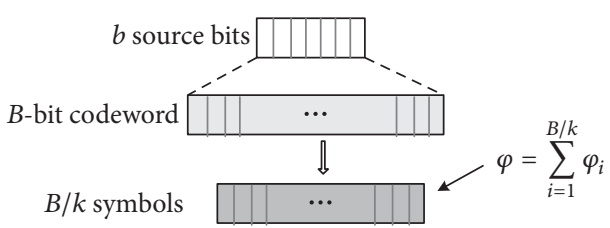

(b) SoftPHY hint calculation for each codeword in coded channel

FIGURE 9: An example of SoftPHY hint calculation under the QPSK modulation. For uncoded channel, the hint $\varphi_{i}$ is calculated as the distance between the received signal $y_{i}$ 's constellation point and the decoded symbol $a_{i}$ 's constellation point. For coded channel where a group of $b$ bits are coded to $B$ codeword, then modulated to $B / k$ symbols, the SoftPHY hint is calculated for this $b$-bit stream, which is the sum of $\varphi_{i}$ for each symbol. The codeword with a larger $\varphi$ value is determined to be detected correctly with a higher probability.

Recitation [28] exploits the SoftPHY-like idea at the transmitter side. The authors observe that the wireless physical layer is deterministic when the processes of signal scrambling, encoding, interleaving, and modulating are known. Thus, before actual transmission, the transmitter can "rehearse" every operation in the decoding process to predict the received bits' confidence, called error event probability (EVP), based on which information, it then takes the best action for the following data transmission through some possible schemes such as unequal error protection, partial packet recovery, and rate adaptation. The authors further propose UnPKT [29] to add more FEC redundancies for the bits with higher estimated EVP in the packet, so as to ensure its decoding at the receiver side.

\subsection{SIC}

4.3.1. Introduction of SIC. The technology of successive interference cancellation (SIC) is one version of the interference cancellation techniques which harness the data structure in the interference to mitigate its harmful effect, thus improving the channel utilization. SIC was first implemented in WLANs since about 2008.

Suppose a received signal $y[n]$ contains two signals $y_{1}[n]$ and $y_{2}[n]$ from different transmitters:

$$
y[n]=y_{1}[n]+y_{2}[n]+w[n],
$$

where $w[n]$ indicates the Gaussian background noise.

Assume the signal strength of $y_{1}$ is much stronger than that of $y_{2}$, and

$$
\operatorname{SINR}_{1}=\frac{y_{1}}{y_{2}+w} \geq \beta,
$$

where $\beta$ is the SINR threshold to demodulate the signal.

In this situation, the signal $y_{1}$ can be detected correctly through the normal demodulation process. The demodulated bit stream for $y_{1}$ can be utilized to reconstruct its received signal $y_{1}^{\prime}$. At this time, the signal $y_{2}$, whose SINR in the received signal $y$ is obviously below $\beta$, can also be detected through subtracting $y_{1}^{\prime}$ from $y$ if the following condition is satisfied:

$$
\operatorname{SINR}_{2}\left(y-y_{1}^{\prime}\right)=\frac{y_{2}}{y-y_{1}^{\prime}+w} \geq \beta .
$$

Figure 10 illustrates an example of the SIC process in a scenario when two signals are collided, and both signals can be detected correctly through this process. The SIC process can be extended to $k(k>2)$ collided packets scenarios theoretically.

4.3.2. Survey of SIC. This technology has been exploited to increase concurrent transmissions or adapt optimal transmission rate.

(i) Avoid Interference and Exploit Concurrency. SIC is introduced in WLANs to mainly increase the channel capacity through enabling more concurrent transmissions. Authors in [5] first implement this technique in WLANs. Figure 11 gives a simple example for its application, where three clients intend to transmit their data packets to the AP. Different from the 802.11 standard which utilizes CSMA to avoid their mutual interference, SIC permits their simultaneous transmissions through carefully adjusting the transmission power to satisfy the SIC decoding requirements. After receiving the collided signal, the AP first decodes the packet 1 with the strongest power and then reconstructs and cancels this packet from the received signal; it then repeats this process to decode packet 2 and packet 3 .

With a different opinion on SIC, authors in [30] investigate the throughput gain of SIC from the MAC layer perspective. They point out that the network performance can be improved through SIC only under some limit conditions for the transmission data rate and the SNR requirements. These conditions are so restrictive and hard to be satisfied, making the gains of SIC not achievable in practical networks.

The idea of SIC has also been employed in [31-33] to improve the network performance through Aloha-based random access, where the transmissions are divided into time slots, and each client randomly selects one slot for its 


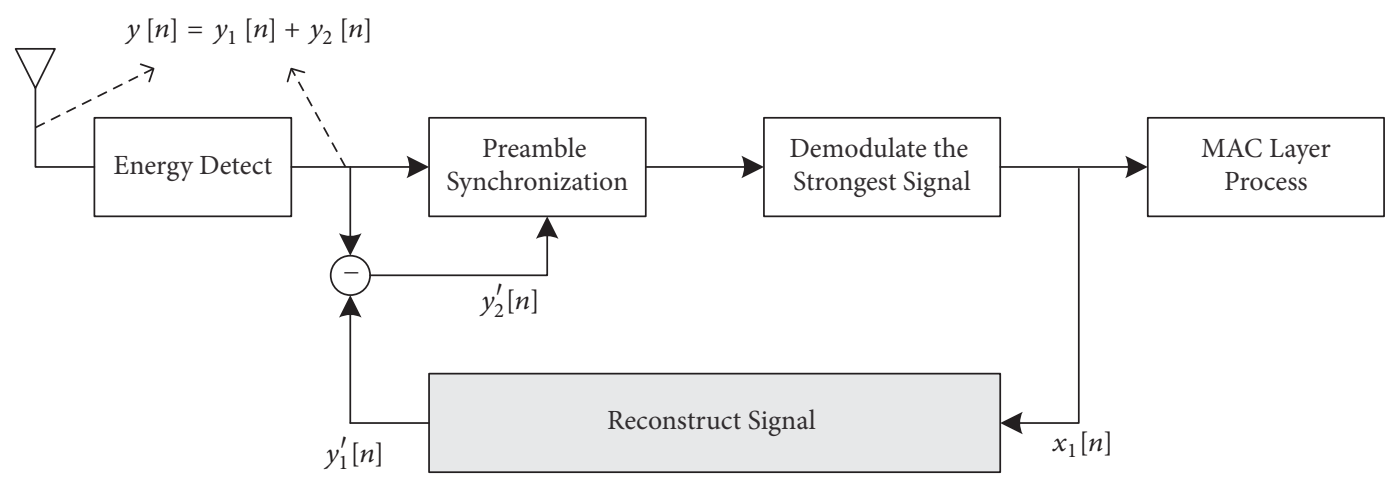

FIGURE 10: An example of SIC process when a node receives two signals $y_{1}[n]$ and $y_{2}[n]$ simultaneously, where the signal strength of $y_{1}[n]$ is much stronger than that of $y_{2}[n]$. The node first demodulates the strongest signal $y_{1}[n]$ and gets the corresponding transmitted bit stream $x_{1}[n]$; it then reconstructs the ideal received signal $y_{1}^{\prime}[n]$ from $x_{1}[n]$ and subtracts $y_{1}^{\prime}[n]$ from $y[n]$ to get the second signal $y_{2}[n] ; y_{2}[n]$ is finally fed into the same preamble synchronization and demodulation process to get the corresponding bit stream $x_{2}[n]$. This scenario can be extended to $k(k>2)$ collided packets scenarios theoretically.

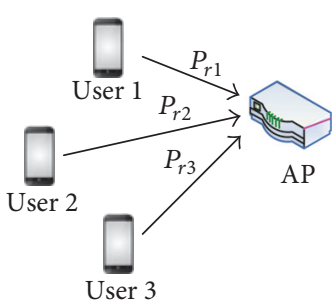

(a) The scenario

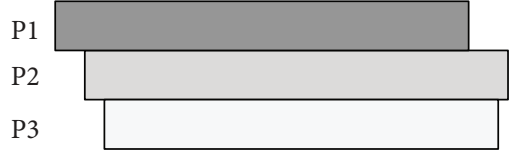

(b) The received packets at $\mathrm{AP}$

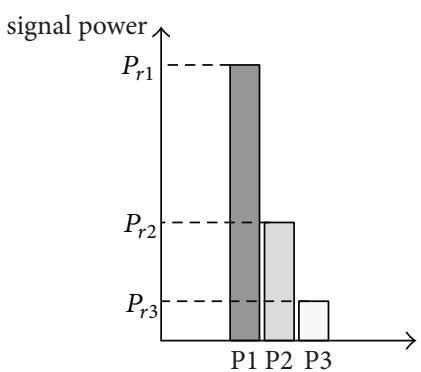

(c) Receiving power comparison

FIgURE 11: A simple example when SIC is applied in WLAN. The three clients transmit their data packets to the AP simultaneously. Through adjusting the transmission power carefully, the receiving power of the three packets $P_{r 1}, P_{r 2}$, and $P_{r 3}$ can satisfy the SIC decoding requirement, making all the three packets decoded successfully.

data transmission. The SIC is applied to permit multiple transmissions in each slot, and the decoding constraints are satisfied through changing the clients' transmission power, data rate, etc.

ContraFlow [34] is a SIC MAC protocol designed for fullduplex wireless networks. Based on the integration of fullduplex and the CSMA/CA in the standard, it designs a duallink which enables two links to be proceeded concurrently to both increase the spatial reuse and eliminate the hidden terminals. Contrabass [35] further exploits this technique in the MIMO system to enable the channel training when a receiver receives multiple packets from different transmitters. CSMA $k$-SIC [36] exploits the SIC in the distributed random access protocols and makes a receiver capable of cancelling up to $k$ strongly interfering signals, through determining which links can be scheduled and which interferers a receiver must cancel.

BASIC [37] applies this technique in the enterprise WLANs. It utilizes the AP backbone to design an uplink transmission strategy, which permits simultaneous transmissions from multiple clients to APs and controls the data rates of the clients so as to collaboratively exploit the SIC mechanism at the APs to decode all the packets, through utilizing the diversity of received signal across multiple receivers. (ii) Rate Adaptation. The basic concept of SIC has also been exploited with rateless coding to further improve the network performance, such as Strider [6] and AutoMAC [38], which will be discussed in the following part.

\subsection{Rateless Coding}

4.4.1. Introduction of Rateless Coding. Rateless coding has been introduced for a long time, starting from the automatic repeat request (ARQ) schemes. Its objective is to make the signals with fixed channel coding and modulation schemes decoded at any SNR environments. The first kind of practical rateless codes is LT codes [39], which is designed for the erasure channel where the transmission packets has some probability to be lost. Raptor codes [40] are later introduced to achieve a more computational efficient and capacity achieving performance. For the AWGN channel, a "layered" encoding and decoding manner [41] is proposed to combine the original codes with fixed rate to generate a rateless stream.

One of the main challenges of rateless coding is to reduce the processing complexity to make it more practical [42]. Some current works focus on designing practical rateless codes or the corresponding up-layer protocols and make 


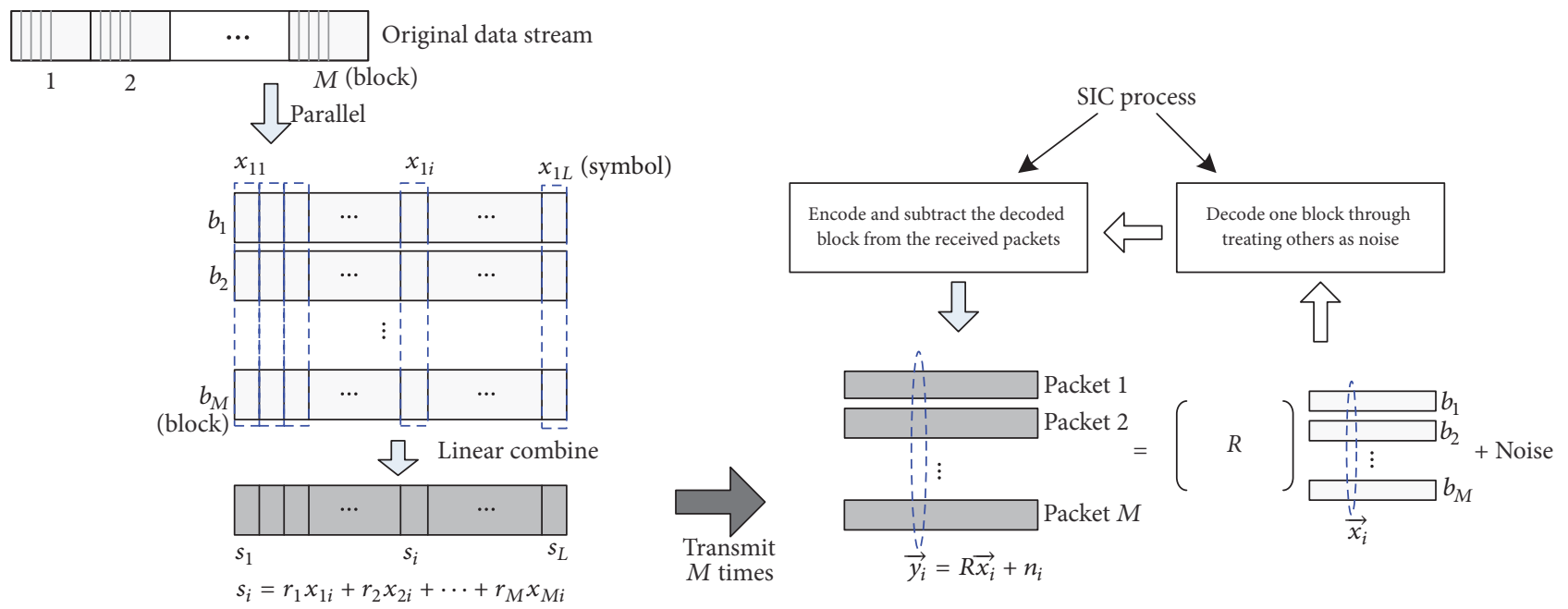

FIGURE 12: The rateless coding scheme in Strider. The original data stream is divided into $M$ blocks, each with $L$ complex symbols. The $M$ blocks are linearly combined to form a new packet for transmission. The sender will create multiple packets using different linear combinations and transmit them until the receiver can decode all the $M$ blocks. After receiving enough signals, the receiver first decodes the first block through treating the other blocks as noise, then reencodes it, and subtracts it from the received signal to proceed the next block. It will repeat this process until all the blocks are decoded.

implementations on the system testbeds to evaluate their performance.

4.4.2. Survey of Rateless Coding. The aim of rateless coding is to achieve optimal transmission rate under any wireless environments.

Strider [6] is the first work that exploits rateless coding in the WLAN-like scenarios and has been implemented on system testbed. It designs a rateless coding technique and combines it with SIC to permit concurrent transmissions of multiple coded streams, thus achieveing an optimal data rate in any SNR situation. As shown in Figure 12, the original data stream is divided into $M$ blocks, each block with $L$ complex symbols. The $M$ blocks are linearly combined to form a new packet for transmission. The sender will create multiple packets using different linear combinations and transmit them until the receiver can decode all the $M$ blocks. After receiving these packets, the receiver first decodes the first block through treating the other blocks as noise and then reencodes it and subtracts it from the received signal to proceed the next block. It will repeat this process until all the blocks are decoded. Through this way, the sender can achieve the optimal bit rate without knowing the channel states.

The authors further propose AutoMAC [38], a MAC protocol for Strider to improve its efficiency. In Strider, the number of rateless transmissions of each packet depends on the SNR environments at the receiver side, which information the transmitter does not know. AutoMAC makes the receiver ACK the corresponding transmitter once it determines that it has received enough number of signals to decode the packet. The ACKed transmitter then goes to the next packet transmission, without wasting the channel bandwidth.

Different from linear combination, spinal code [43] exploits a pseudo-hash function to transform the original data bits into encoded symbols. due to the sequential application of the hash function, this encoding process ensures that two similar streams with even one-bit difference have different coded sequences, thus more resilient to noise and bit errors. The authors also exploit the sequential structure of the encoding for the decoding process through a tree-searching method. They demonstrate spinal code's higher throughput and its ability to be deployed practically through hardware prototype.

RateMore [44] is a link-layer protocol design for rateless codes to determine how much data needed to be transmitted, so as to both guarantee the successful data decoding and avoid wasting the transmission time. The sender learns the decoding CDF (cumulative probability distribution function), which represents the probability distribution of the number of symbols required to decode a packet, based on the acknowledgment from the receiver. With both the decoding CDF and the feedback delay, an optimal transmission schedule is derived to maximize the network performance.

PRAC [45] exploits the feature of rateless linear coding to identify and recover the erroneous packet segments in the partial packet recovery context. The transmitter continually makes linear combination of $k$ original packets to create new one for transmission. After receiving $n(n>k)$ packets, the receiver begins to distinguish the erroneous symbols through utilizing a designed scheme called algebraic consistency rule check (ACR). For each symbol column, it iteratively conducts the ACR check, a searching algorithm to identify correct symbols, and conducts the CRC check until the ACR check is satisfied. This mechanism reduces the retransmitted segments, thus significantly improving the transmission efficiency.

4.5. Physical Unique Features. In this part, we will survey a series of features in the OFDM modulation process exploited 


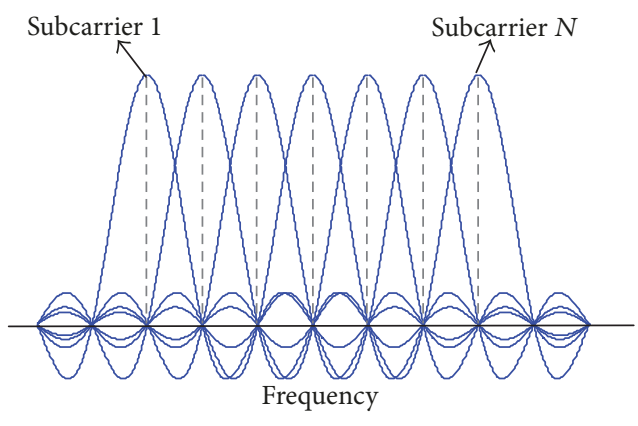

(a) OFDM spectrum

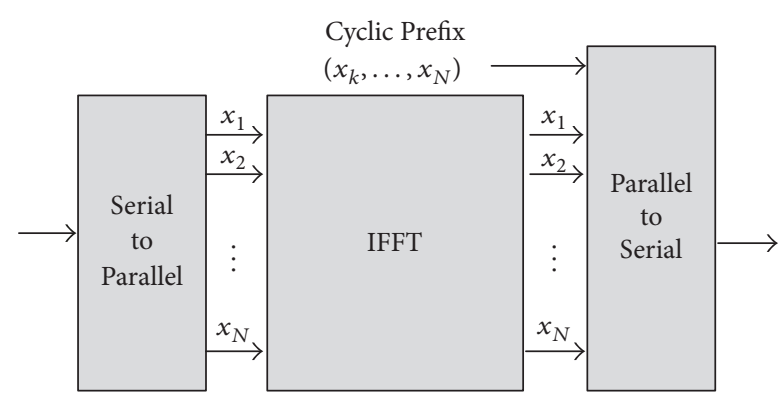

(b) OFDM modulation

FIgURE 13: The OFDM modulation and spectrum.

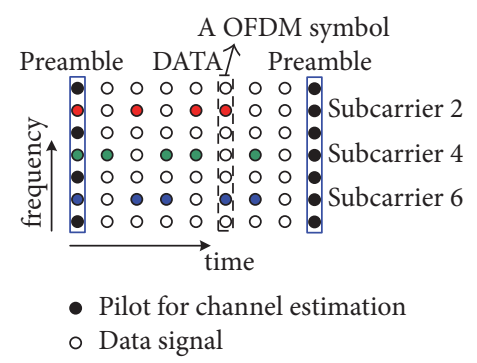

(a) An example of attached information in hJam

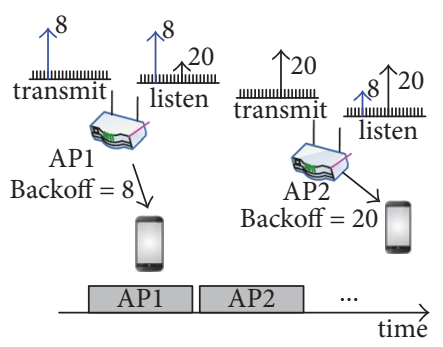

(b) An example of Back2F

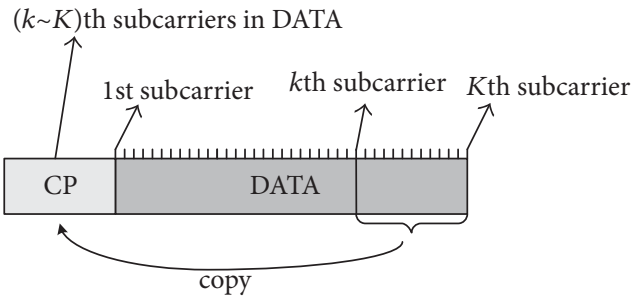

(c) The feature of cycle prefix exploited by CPRecycle

FIGURE 14: Three interference management schemes through exploiting the OFDM features.

for interference management; meanwhile, some features in other systems are also concluded.

4.5.1. Introduction of OFDM System. As shown in Figure 2, OFDM is part of the digital modulation and demodulation process in current 802.11 standard. An OFDM signal can be described as a group of closely spaced modulated subcarriers, which are orthogonal and will not interfere with each other theoretically, as demonstrated in Figure 13(a). The OFDM modulation process is shown in Figure 13(b); the modulated data are first passed through the serial-to-parallel $(\mathrm{S} / \mathrm{P})$ module to map the data in each subcarrier and then fed into IFFT (Inverse Fast Fourier Transform) to output the timedomain OFDM symbol. Cyclic Prefix is inserted in this stage to eliminate the intersymbol interference. The signal will finally be output as a serial stream for the following process.

4.5.2. Survey on Exploiting OFDM Features. To manage interference efficiently, some known OFDM features are exploited to avoid interference, increase concurrent transmissions, or reduce the coordination overhead.

(i) Avoid Interference and Exploit Concurrency. hJam [46] makes the control information attached to the data packet for transmission, so as to reduce its transmission overhead. It exploits a few of "clean" subcarriers which have no signal except noise in the packet preamble to carry the control information. It then proposes attachment coding to make the control information transmitted in these "clean" subcarriers of the data packet. As shown in Figure 14(a), the clean subcarriers are 2, 4, and 6, and the control information attached in subcarrier 2 is 0101100 . The receiver can determine there is an attached signal on a subcarrier if it detects a relatively high level energy on that subcarrier. After detecting the attached signals, node can obtain the corresponding control messages. Meanwhile, the data signal can be recovered through detaching the attached control signal from the received signal. The authors further propose Attached-RTS [47] and FAST [48] to solve the hidden terminal and exposed terminal problems through exploiting the attached control information.

CPRecycle [49] exploits the redundancy in cycle prefix of the OFDM PHY to mitigate interference. As shown in Figure 14(c), the cycle prefix is a copy of a portion of the following symbol in the tail, and it is used to eliminate the intersymbol interference induced by the multipath propagation in the wireless channel. It is now well known to be overprovisioned. For example, the 802.11 recommends the CP duration to be $0.8 \mu \mathrm{s}$ for $20 \mathrm{MHz}$ bandwidth, but in most cases the multipath delay is only in the order of $n s$ [50]. The authors get a key observation that when a receiver performs FFT from different positions in the redundancy cyclic prefix, the signal demodulation will not be affected but the interference from concurrent transmissions can be dramatically reduced. They then design an algorithm to find the optimal starting position to maximize the performance.

(ii) Reduce Coordination Overhead. T2F [51] and Back2F [52] propose to reduce the coordination overhead induced by the 
backoff, through migrating the random backoff from time domain to frequency domain. Each node needs two antennas, one for data transmissions and the other for listening to all the subcarriers in the network. Before the transmission, each node selects a subcarrier and transmits signals in this subcarrier, while the subcarrier number indicates its backoff time. The node with the minimal subcarrier number wins the contention and transmits the data packet. As shown in Figure 14(b), when both AP1 and AP2 intend to transmit packets to their clients, AP1 wins the contention as its backoff value is 8 . AP2 will transmit its packet immediately after AP1's transmission, as it has the subminimal backoff value. The authors also propose multiple contention rounds to combat some situations such as multiple contention domains. Different from T2F [51] and Back2F [52] which only use subcarriers to reduce the backoff time, REPICK [53] further exploits the OFDM subcarriers to reduce some other overhead induced by 802.11 MAC, including DIFS, and ACK; thus it can dramatically improve the network performance.

4.5.3. Survey on Other Systems. Some strategies exploit the known feature in other systems to reduce the coordination overhead and enable concurrent transmissions. Side Channel $[54,55]$ utilizes the Direct-Sequence Spread Spectrum (DSSS) system which has the ability to resist interferences to a certain extent [56]. The authors carefully design some "intended patterns" which carry the control information and make them transmitted simultaneously with the original data packet, so as to reduce the coordination overhead but without degrading the effective throughput of data transmissions. Coco [57] advocates simultaneous accesses from multiple senders to a shared channel, optimistically allowing collisions instead of simply avoiding them, through both utilizing the capture effect and exploiting the ability to tolerate collisions because of redundancy in the physical layer implementations. mZig [58] exploits the known shaping feature of the ZigBee physical layer design. Based on this shaping feature, it can resolve one $m$-packet collision by this collision itself and thus can achieve $m$-fold throughput improvement comparing with the legacy mechanisms.

Some strategies exploit the physical layer features to improve the retransmission efficiency. MISC [59] merges incorrect symbols from multiple transmissions to produce correct ones. It exploits constellation diversity by rearranging the constellation maps in retransmissions, so as to improve the combining and decoding efficiency at the receiver side. Epicenter [60] utilizes the backbone network in enterprise WLANs, where one packet transmitted by a client will be received by multiple APs and shared among each other. They exploit the multiple copies of the incorrectly received symbols to recover the transmitted signal and design a coarse representation of the symbols to reduce their overhead when transmitted between APs.

\section{Discussion}

The aim of the surveyed paper is to exploit the physical layer techniques to manage interference in wireless networks efficiently, so as to improve the network throughput. This part discusses the challenges of applying these techniques and gives some potential future directions for research in this area.

5.1. Discussions of the Physical Layer Techniques. The adoption of the physical layer techniques inspires new ways for designing high efficient channel access mechanisms. Here we want to discuss the advantages and challenges of these techniques as a summary.

5.1.1. Cross Correlation. Cross correlation can be exploited to detect both the control and data packets once a collision occurs. When this technology is utilized to recover the collided control packets, the coordination information can be exchanged among nodes more efficiently, thus avoiding interference, enabling concurrent transmissions, or reducing the coordination overhead. When this technology is utilized to recover the collided data packets, nodes have a new way to manage interference through embracing it but not avoiding it.

The main challenge of applying the cross correlation technology for interference management lies in the high computational overhead. According to Section 4.1.1., one cross correlation process needs $L$ complex multiplication calculations. When leveraging this technology to recover the collided control packet and suppose the number of possible known sequences carried by the control packet is $N$, one node should conduct $N$ cross correlation processes at each position of the received signal to determine which known sequence is detected $[13,22,23]$; this will lead to $N \times L$ complex multiplication calculations at each position. The computational overhead would be even higher when this technology is exploited to recover the collided data packets $[18,19]$, as the cross correlation should be conducted for multiple times when bootstrapping the packet decoding. None of the works has been implemented on off-the-shelf devices till now, and all of them are evaluated based on special platforms such as USRP [61] and WARP [62].

5.1.2. SoftPHY. SoftPHY provides PHY's confidence of each bit to the upper layer, so as to either increase the retransmission efficiency through only making the bits with lowconfidence retransmitted or adapt the optimal transmission rate through calibrating the channel estimation.

The main problem of SoftPHY is its low performance when applied in poor wireless environments. SoftPHY relies on preamble to synchronize with the incoming signal and estimate the confidence of the following bits. However, under the situation of interference or comparatively high noise, the receiver would not be able to detect the preamble correctly, making the following bits decoding and confidence calculation not achievable. Authors in PPR [4] utilize a postamble at the end of each packet to make SoftPHY work when the preamble is corrupted. However, this design would not be practical in real networks as all parts of a packet may have a high probability to be corrupted when the wireless channel is poor.

5.1.3. SIC. SIC permits concurrent transmissions of multiple packets and detects them correctly when the receiving power 
satisfies the constraints. In the SIC receiving process, one node first decodes the strongest signal and then subtracts it and decodes the inferior signal if the remaining SINR is above the threshold; the process can be repeated to decode more signals.

The practical hurdle of deploying SIC in wireless networks is how to satisfy the power constraints. Although some advances try to exploit SIC to improve the network throughput in a distributed way [34-36], it is intuitively very hard to enable multiple concurrent transmissions and make their receiving power satisfy the restrictive requirements through protocol design. Actually, some researchers have already investigated the throughput gain of SIC from the MAC layer perspective [30] and pointed out that the throughput gain can only be obtained under limit conditions for the transmission data rate and the SNR requirements, which are very hard to be satisfied in practical networks. Centralized coordination may be a better choice for the SIC application, as proposed by Basic [37] which utilizes the AP backbone to centrally control the data rate and transmission power of simultaneous transmissions.

5.1.4. Rateless Coding. Rateless coding makes the undecodable packet in a fixed data rate be recovered by combining multiple transmissions, resulting in different data transmission rate. Comparing with the traditional rate adaptation mechanisms which choose the best data rate through the estimated SNR of the wireless channel, this method can achieve the optimal bit rate without knowing the channel state in advance.

One of the main challenges of rateless coding is to reduce its processing complexity to make it more practical. Some current advances have focused on this challenge and designed mechanisms with affordable computational complexity for implementation, such as Strider [6] and spinal codes [43]. However, these kinds of mechanisms incur significant modification in the baseband process as they require the transmitters to transmit correlated symbols, making these mechanisms hard to be embedded to current wireless devices.

5.1.5. Unique Physical Layer Features. Several unique features in the physical layer are exploited for interference management, such as the OFDM subcarriers, redundancy design in both OFDM and DSSS systems.

The OFDM subcarriers are exploited to convey the coordination information, so as to effectively solve the hidden or exposed terminal problem $[47,48]$, or to reduce the coordination overhead induced by backoff [52]. It is a good idea to leverage the OFDM subcarriers to convey the coordination information. However, some of the current works need extra antenna to accomplish the transmission $[48,52]$. Although multiple antennas are commonly deployed in current wireless devices due to the adoption of MIMO (multi-input and multioutput) technology in recent 802.11n and 802.11ac standards, it is still uneconomical to let one antenna only transmit the control information.

Coding redundancy in both OFDM and DSSS systems is originally designed for interference resistance, so that the signals can be decoded in comparatively lower SINR environments. When the control information is transmitted simultaneously with the data packets, it becomes an interference of the data packet and will reduce the data packet's received SINR, making the system have lower ability to resist other interference. Thus, these mechanisms will only work well in the high SNR environments.

5.1.6. Summary of Limitations. Based on the above discussions, we summarize the main limitations to deploy these physical layer techniques to real networks as follows:

(i) High computational overhead: the deploying of each physical layer technique will certainly induce extra computational overhead to the systems, which has become the main hurdle to make them applicable, such as cross correlation and rateless coding. Most of the works have just been evaluated based on special software defined radio platforms which have huge computation resources; few of them can be implemented on commercial devices.

(ii) Rigorous system constraints: the successful application of some physical layer techniques can only occur under rigorous constraints. For example, SoftPHY requires uncollided preamble for synchronization and SIC has strict power constraints for the multiple receiving packets. These requirements can hardly be fully satisfied in real networks, especially the mobility scenarios when the infrastructures (APs) and stations are mobile, making the interference and receiving power more uncertain in the network. This issue would significantly interrupt the performance improvement of the proposed mechanisms.

(iii) Hardware incompatibility: nearly all the physical layer techniques have added or revised some components in the baseband signal process, making them incompatible to current commercial devices.

\subsection{Discussions of Future Directions}

5.2.1. Overcome the Limitations of Physical Layer Techniques. As discussed above, the current mechanisms based on exploiting the physical layer techniques have some serious limitations which impede their applications, such as the high computational overhead, the rigorous system constraints, and hardware incompatibility. Overcoming these limitations would obviously enhance their deploy ability in real networks. For example, the hardware compatibility is now becoming an important specification to evaluate the proposed mechanisms, and this issue has been considered by many current works based on physical layer design, such as WeBee [63]. It is worth rethinking the mechanism design from this point of view.

5.2.2. Further Explore the Physical Layer Techniques. Current advances have already presented some examples on exploiting the physical layer techniques for interference management and demonstrated exciting throughput improvement through hardware experiments or simulations. However, we consider the potential of these techniques in these areas is far 
from fully unleashed. Researchers may find some other effective methods to improve the network performance through utilizing these physical layer techniques. For example, SIC has been exploited only to enable concurrent transmissions of data packets, how to design the interference management mechanism when it is utilized for concurrent transmissions of data and control packets, and what is the expected performance?

5.2.3. Manage Interference Centrally. Researchers have observed that some of the problems in the legacy interference management, such as the exposed and hidden terminals and the large coordination overhead, are intrinsic properties existing in the distributed coordination methods [10]. That means optimizing CSMA or designing new distributed interference management mechanisms can only mitigate these problems but not eliminate them. Actually, the 802.11 standard already recommends a centralized coordination mechanism, called PCF (point coordination function), to make the AP schedule the uplink and downlink transmissions centrally to achieve a higher throughput. However, this mechanism is rarely used in current WLANs as it is not suitable to a common scenario when there are multiple APs in the network.

Nowadays, many researchers focus on centralized coordination mechanisms through exploiting the wired backbone network among APs in the enterprise WLANs. For example, Symphony [19] extends the idea of Zigzag [18] in the multiple-AP scenario through utilizing the AP backbone; Basic [37] also utilizes this backbone to control the data rate and simultaneous transmissions in the uplink direction and exploits SIC at APs to decode all the packets. Designing centralized interference management mechanisms seems like a more promising way to achieve high performance in wireless networks. This concept can also be extended for designing efficient rate adaptation and retransmission schemes. In addition, when considering the infrastructure mobility scenario with multiple mobile APs but without the backbone network, how to solve this kind of issues is a very meaningful topic.

5.2.4. Extend the Design to MIMO. Current researches on interference management through exploiting physical layer techniques mainly focus on the single stream systems; only very few of them are designed or tested based on the MIMO systems, such as Recitation [28]. Actually, there have already been some researches on theoretically analyzing the performance of exploiting these physical layer techniques to the MIMO communications, for example, exploiting rateless coding for MIMO Fading Channels [64] and proposing SIC for large-scale MIMO configuration [65] and 5G system [66]. However, these works are accomplished from the communication perspective and they do not consider the suitable design of channel access mechanisms in the network. It would be worthy of expectation to exploit these physical layer techniques in the MIMO systems.

\section{Conclusion}

In this paper, we investigate the interference management mechanisms which exploit the physical layer techniques in wireless networks. We first give some background information about the PHY and MAC characteristics in the 802.11 standards and then discuss the problems existing in the current interference management process. After that, we introduce five kinds of physical layer techniques and investigate how these techniques are exploited to manage interference and improve the network throughput. Based on this study, we finally present some discussions from both the physical layer and MAC layer perspective. We hope this survey would help the readers to summarize the current research progress and inspire their future work.

\section{Conflicts of Interest}

The authors declare that they have no conflicts of interest.

\section{Acknowledgments}

This work was supported in part by grants from China NSFC (61702343, 61472259, and 61601308), China Postdoctoral Funding (2017M610548), Joint Key Project of the National Natural Science Foundation of China (U1736207), Guangdong Natural Science Foundation (2017A030312008), Hong Kong RGC (PolyU-521312), Hong Kong PolyU (4-BCB6, MN020, G-YBXY), Shenzhen Science and Technology Funding (JCYJ20170302140946299 and JCYJ20170412110753954), Fok Ying-Tong Education Foundation for Young Teachers in the Higher Education Institutions of China (161064), Guangdong Talent Project (2014TQ01X238 and 2015TX01X111), and GDUPS (2015). Kaishun Wu is the corresponding author.

\section{References}

[1] J. Xu, J. Yao, L. Wang, Z. Ming, K. Wu, and L. Chen, "Narrowband Internet of Things: Evolutions, Technologies and Open Issues," IEEE Internet of Things Journal, 2017.

[2] Cisco, Cisco Visual Networking Index: global mobile data traffic forecast update, 2016 - 2021 white paper, 2017.

[3] K. Tan, J. Fang, Y. Zhang et al., "Fine-grained channel access in wireless lan," in Proceedings of the ACM SIGCOMM Conference (SIGCOMM '10), pp. 147-158, New Delhi, India, August 2010.

[4] K. Jamieson, The SoftPHY abstraction: From packets to symbols in wireless network design [Ph.D. thesis], MIT, 2008.

[5] D. Halperin, T. Anderson, and D. Wetherall, "Taking the sting out of carrier sense: interference cancellation for wireless LANs," in Proceedings of the 14th ACM Annual International Conference on Mobile Computing and Networking (MobiCom '08), pp. 339-350, September 2008.

[6] A. Gudipati and S. Katti, "Automatic rate adaptation and collision handling," Computer Communication Review, vol. 41, no. 4, pp. 158-169, 2011.

[7] A. P. Jardosh, K. N. Ramachandran, K. C. Almeroth, and E. M. Belding-Royer, "Understanding congestion in IEEE 802.11b wireless networks," in Proceedings of the 5th ACM SIGCOMM Conference on Internet Measurement (IMC '05), October 2005.

[8] Y. He, R. Yuan, J. Sun, and W. Gong, "Semi-random backoff: towards resource reservation for channel access in wireless LANs," in Proceedings of the 17th IEEE International Conference on Network Protocols (ICNP '09), pp. 21-30, IEEE, Princeton, NJ, USA, October 2009. 
[9] M. Vutukuru, K. Jamieson, and H. Balakrishnan, "Harnessing exposed terminals in wireless networks," in Proceedings of the ACM NSDI, 2008.

[10] Z. Yang, J. Zhang, K. Tan, Q. Zhang, and Y. Zhang, "Enabling TDMA for today's wireless LANs," in Proceedings of the IEEE Conference on Computer Communications (INFOCOM '15), pp. 1436-1444, Kowloon, Hong Kong, April 2015.

[11] L. Wang, X. Qi, J. Xiao, K. Wu, M. Hamdi, and Q. Zhang, "Exploring Smart Pilot for Wireless Rate Adaptation," IEEE Transactions on Wireless Communications, vol. 15, no. 7, pp. 4571-4582, 2016.

[12] S. Sen and N. Santhapuri, "Moving away from collision avoidance: towards collision detection in wireless networks," in Proceedings of the ACM HotNets, Moving away from collision avoidance, 2009.

[13] S. Sen, R. R. Choudhury, and S. Nelakuditi, "CSMA/CN: carrier sense multiple access with collision notification," in Proceedings of the 16th Annual Conference on Mobile Computing and Networking (MobiCom '10), pp. 25-36, Chicago, Ill, USA, September 2010.

[14] T. Xiong, J. Zhang, J. Yao, and W. Lou, "Symbol-level detection: A new approach to silencing hidden terminals," in Proceedings of the 20th IEEE International Conference on Network Protocols, ICNP '12, USA, November 2012.

[15] E. Magistretti, O. Gurewitz, and E. W. Knightly, "802.1lec: collision avoidance without control messages," in Proceedings of the 18th Annual International Conference on Mobile Computing and Networking (MobiCom '12), pp. 65-76, ACM, Istanbul, Turkey, August 2012.

[16] J. Yao, T. Xiong, J. Zhang, and W. Lou, "On Eliminating the Exposed Terminal Problem Using Signature Detection," IEEE Transactions on Mobile Computing, vol. 15, no. 8, pp. 2034-2047, 2016.

[17] J. Yao, W. Lou, C. Yang, and K. Wu, "Efficient interference-aware power control in wireless ad hoc networks," in Proceedings of the IEEE International Conference on Communications (ICC '17), pp. 1-6, Paris, France, May 2017.

[18] S. Gollakota and D. Katabi, "Zigzag decoding: combating hidden terminals in wireless networks," in Proceedings of the ACM SIGCOMM Conference on Data Communication (SIGCOMM '08), pp. 159-170, August 2008.

[19] T. Bansal, B. Chen, P. Sinha, and K. Srinivasan, "Symphony: Cooperative packet recovery over the wired backbone in enterprise WLANs," in Proceedings of the 19th Annual International Conference on Mobile Computing and Networking, MobiCom '13, pp. 351-362, USA, October 2013.

[20] X. Zhang and K. G. Shin, "DAC: Distributed asynchronous cooperation for wireless relay networks," in Proceedings of the IEEE INFOCOM '10, USA, March 2010.

[21] X. Zhang and K. G. Shin, "Chorus: collision resolution for efficient wireless broadcast," in Proceedings of the IEEE International Conference on Computer Communications (INFOCOM '10), pp. 1-9, San Diego, Calif, USA, March 2010.

[22] S. Misra and M. Khatua, "Semi-distributed backoff: collisionaware migration from random to deterministic backoff," IEEE Transactions on Mobile Computing, vol. 14, no. 5, pp. 1071-1084, 2015.

[23] J. Zhao, S. Fan, D.-A. Li, and B. Zhao, "Collision tolerance: Improving channel utilization with correlatable symbol sequences in wireless networks," International Journal of Distributed Sensor Networks, vol. 2015, Article ID 678735, 2015.
[24] K. Jamieson and H. Balakrishnan, "PPR: partial packet recovery for wireless networks," in Proceedings of the ACM SIGCOMM Conference on Computer Communications '07, pp. 409-420, Japan, August 2007.

[25] M. Vutukuru, H. Balakrishnan, and K. Jamieson, "Crosslayer wireless bit rate adaptation," in Proceedings of the ACM SIGCOMM conference '09, Barcelona, Spain, August 2009.

[26] S. Sen, N. Santhapuri, R. R. Choudhury, and S. Nelakuditi, "AccuRate: constellation based rate estimation in wireless networks," in Proceedings of the NSDI, 2010.

[27] M. O. Khan, L. Qiu, A. Bhartia, and K. C. Lin, "Smart Retransmission and Rate Adaptation in WiFi," in Proceedings of the 23rd IEEE International Conference on Network Protocols (ICNP '15), pp. 54-65, San Francisco, CA, USA, November 2015.

[28] Z. Li, Y. Xie, M. Li, and K. Jamieson, "Recitation: Rehearsing wireless packet reception in software," in Proceedings of the 21st Annual International Conference on Mobile Computing and Networking, MobiCom '15, pp. 291-303, Paris, France, September 2015.

[29] Y. Xie, Z. Li, M. Li, and K. Jamieson, "Augmenting wideband 802.11 transmissions via unequal packet bit protection," in Proceedings of the 35th Annual IEEE International Conference on Computer Communications, IEEE INFOCOM '16, USA, April 2016.

[30] S. Sen, N. Santhapuri, R. R. Choudhury, and S. Nelakuditi, "Successive interference cancellation," in Proceedings of the the Ninth ACM SIGCOMM Workshop, pp. 1-6, Monterey, California, October 2010.

[31] S. P. Weber, J. G. Andrews, X. Yang, and G. de Veciana, “Transmission capacity of wireless ad hoc networks with successive interference cancellation," Institute of Electrical and Electronics Engineers Transactions on Information Theory, vol. 53, no. 8, pp. 2799-2814, 2007.

[32] J. Blomer and N. Jindal, "Transmission capacity of wireless ad hoc networks: Successive interference cancellation vs. joint detection," in Proceedings of the 2009 IEEE International Conference on Communications, ICC '09, Dresden, Germany, June 2009.

[33] M. Mollanoori and M. Ghaderi, "On the performance of successive interference cancellation in random access networks," in Proceedings of the 9th Annual IEEE Communications Society Conference on Sensor, Mesh and Ad Hoc Communications and Networks, SECON '12, pp. 461-469, Seoul, South Korea, June 2012.

[34] N. Singh, D. Gunawardena, A. Proutiere, B. Radunović, H. V. Balan, and P. Key, "Efficient and fair MAC for wireless networks with self-interference cancellation," in Proceedings of the International Symposium of on Modeling and Optimization of Mobile, Ad Hoc, and Wireless Networks (WiOpt '11), pp. 94-101, IEEE, Princeton, NJ, USA, May 2011.

[35] S. Yoon, I. Rhee, B. C. Jung, B. Daneshrad, and J. H. Kim, “Contrabass: Concurrent transmissions without coordination for ad hoc networks," in Proceedings of the IEEE INFOCOM, pp. 11341142, China, April 2011.

[36] A. Sankararaman and F. Baccelli, "CSMA k-SIC - A class of distributed MAC protocols and their performance evaluation," in Proceedings of the 34th IEEE Annual Conference on Computer Communications and Networks, IEEE INFOCOM '15, pp. 20022010, Kowloon, Hong Kong, May 2015. 
[37] W. Zhou, T. Das, L. Chen, K. Srinivasan, and P. Sinha, "BASIC: backbone-assisted successive interference cancellation," in Proceedings of the the 22nd Annual International Conference, ACM MOBICOM '16, pp. 149-161, New York, NY, USA, October 2016.

[38] A. Gudipati, S. Pereira, and S. Katti, "AutoMAC: rateless wireless concurrent medium access," in Proceedings of the 18th annual international conference on Mobile computing and networking, ACM MOBICOM '12, Istanbul, Turkey, 2012.

[39] M. Luby, "LT codes", in Proceedings of the 43rd Annual IEEE Symposium on Foundations of Computer Science, pp. 271-280, IEEE, Vancouver, Canada, November 2002.

[40] A. Shokrollahi, "Raptor codes," Institute of Electrical and Electronics Engineers Transactions on Information Theory, vol. 52, no. 6, pp. 2551-2567, 2006.

[41] U. Erez, M. D. Trott, and G. W. Wornell, "Rateless coding for Gaussian channels," Institute of Electrical and Electronics Engineers Transactions on Information Theory, vol. 58, no. 2, pp. 530547, 2012.

[42] F. Mehran, K. Nikitopoulos, P. Xiao, and Q. Chen, "Rateless wireless systems: Gains, approaches, and challenges," in Proceedings of the IEEE China Summit and International Conference on Signal and Information Processing, ChinaSIP '15, pp. 751-755, China, July 2015.

[43] J. Perry, P. A. Iannucci, K. E. Fleming, H. Balakrishnan, and D. Shah, "Spinal codes," in Proceedings of the ACM SIGCOMM Conference Applications, Technologies, Architectures, and Protocols for Computer Communication, SIGCOMM '12, pp. 49-60, Helsinki, Finland, August 2012.

[44] P. Iannucci, J. Perry, H. Balakrishnan, and D. Shah, "No symbol left behind: A link-layer protocol for rateless codes," in Proceedings of the International Conference on Mobile Computing and Networking, pp. 17-27, 2012.

[45] G. Angelopoulos, M. Medard, and A. P. Chandrakasan, "Harnessing Partial Packets in Wireless Networks: Throughput and Energy Benefits," IEEE Transactions on Wireless Communications, vol. 16, no. 2, pp. 694-704, 2017.

[46] K. Wu, H. Li, . Lu Wang et al., "HJam: Attachment transmission in WLANs," in Proceedings of the IEEE INFOCOM - IEEE Conference on Computer Communications, pp. 1449-1457, Orlando, FL, USA, March 2012.

[47] L. Wang, K. Wu, and M. Hamdi, "Attached-RTS: Eliminating an exposed terminal problem in wireless networks," IEEE Transactions on Parallel and Distributed Systems, vol. 24, no. 7, pp. 12891299, 2013.

[48] L. Wang, K. Wu, and M. Hamdi, "Combating hidden and exposed terminal problems in wireless networks," IEEE Transactions on Wireless Communications, vol. 11, no. 11, pp. 42044213, 2012.

[49] S. Rathinakumar, B. Radunovic, and M. K. Marina, "CPRecycle: Recycling cyclic prefix for versatile interference mitigation in OFDM based wireless systems," in Proceedings of the 12th ACM Conference on Emerging Networking Experiments and Technologies, ACM CoNEXT '16, pp. 67-81, USA, December 2016.

[50] C.-P. Lim, J. L. Volakis, K. Sertel, R. W. Kindt, and A. Anastasopoulos, "Indoor propagation models based on rigorous methods for site-specific multipath environments," IEEE Transactions on Antennas and Propagation, vol. 54, no. 6, pp. 1718-1725, 2006.

[51] S. Sen, R. R. Choudhury, and S. Nelakuditi, "Listen (on the frequency domain) before you talk," in Proceedings of the the
Ninth ACM SIGCOMM Workshop, pp. 1-6, Monterey, California, October 2010.

[52] S. Sen, R. Roy Choudhury, and S. Nelakuditi, "No time to countdown: Migrating backoff to the frequency domain," in Proceedings of the 17th Annual International Conference on Mobile Computing and Networking, MobiCom'11 and Co-Located Workshops, pp. 241-252, USA, September 2011.

[53] X. Feng, J. Zhang, Q. Zhang, and B. Li, "Use your frequency wisely: Explore frequency domain for channel contention and ACK," in Proceedings of the IEEE Conference on Computer Communications, INFOCOM '12, pp. 549-557, USA, March 2012.

[54] K. Wu, H. Tan, Y. Liu, J. Zhang, Q. Zhang, and L. Ni, "Side channel: Bits over interference," in Proceedings of the Sixteenth Annual International Conference, ACM MOBICOM '10, pp. 1324, Chicago, Illinois, USA, September 2010.

[55] K. Wu, H. Tan, Y. Liu, J. Zhang, Q. Zhang, and L. M. Ni, "Side channel: Bits over interference," IEEE Transactions on Mobile Computing, vol. 11, no. 8, pp. 1317-1330, 2012.

[56] K. Wu, H. Tan, H.-L. Ngan, Y. Liu, and L. M. Ni, "Chip error pattern analysis in IEEE 802.15.4," IEEE Transactions on Mobile Computing, vol. 11, no. 4, pp. 543-552, 2012.

[57] X. Ji, Y. He, J. Wang et al., "On Improving Wireless Channel Utilization: A Collision Tolerance-Based Approach," IEEE Transactions on Mobile Computing, vol. 16, no. 3, pp. 787-800, 2017.

[58] L. Kong and X. Liu, "MZig: enabling multi-packet reception in ZigBee," in Proceedings of the 21st Annual International Conference on Mobile Computing and Networking (MobiCom '15), pp. 552-565, Paris, France, September 2015.

[59] J. Ou, Y. Zheng, and M. Li, "MISC: Merging incorrect symbols using constellation diversity for 802.11 retransmission," in Proceedings of the 33rd IEEE Conference on Computer Communications, IEEE INFOCOM '14, pp. 2472-2480, Canada, May 2014.

[60] M. Gowda, S. Sen, R. R. Choudhury, and S.-J. Lee, "Cooperative packet recovery in enterprise WLANs," in Proceedings of the 32nd IEEE Conference on Computer Communications, IEEE INFOCOM '13, pp. 1348-1356, Italy, April 2013.

[61] Ettus Inc, Universal software radio peripheral.

[62] "WARP: Wireless Open Access Research Platform," https:// warpproject.org/trac.

[63] Z. Li and T. He, "WEBee," in Proceedings of the the 23rd Annual International Conference, pp. 2-14, Snowbird, Utah, USA, October 2017.

[64] F. Yijia, L. Lifeng, E. Erkip, and H. V. Poor, "Rateless coding for MIMO fading channels: performance limits and code construction," IEEE Transactions on Wireless Communications, vol. 9, no. 4, pp. 1288-1292, 2010.

[65] A. Elghariani and M. Zoltowski, "Successive interference cancellation for large-scale MIMO OFDM," in Proceedings of the IEEE International Conference on Electro/Information Technology, EIT '15, pp. 657-661, USA, May 2015.

[66] R. Ruby, S. Zhong, H. Yang, and K. Wu, "Enhanced Uplink Resource Allocation in Non-Orthogonal Multiple Access Systems," IEEE Transactions on Wireless Communications, 2017. 


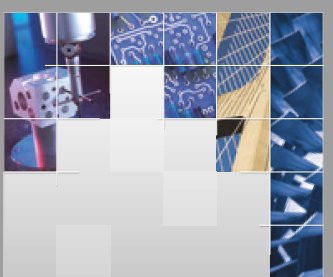

\section{Enfincering}
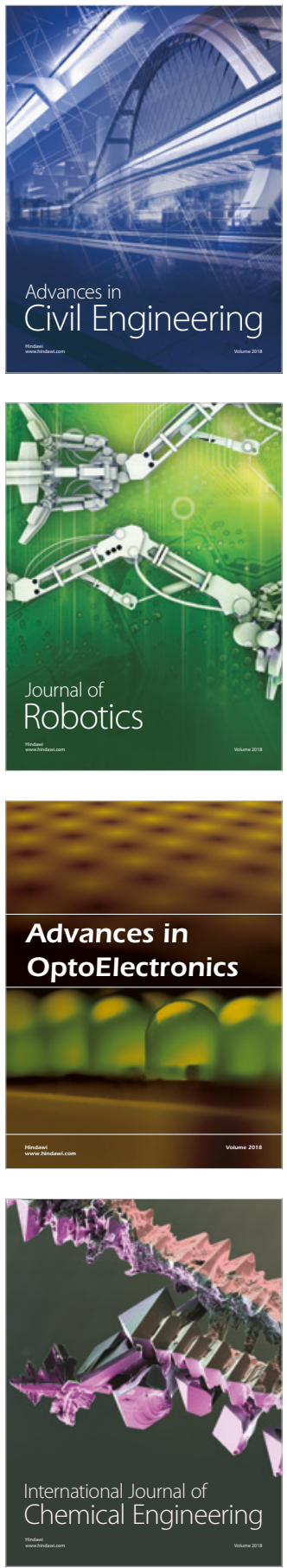

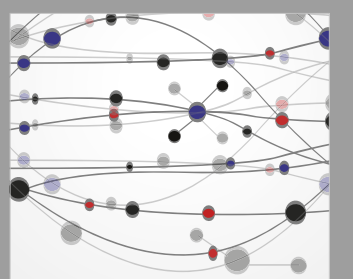

\section{Rotating \\ Machinery}

The Scientific World Journal

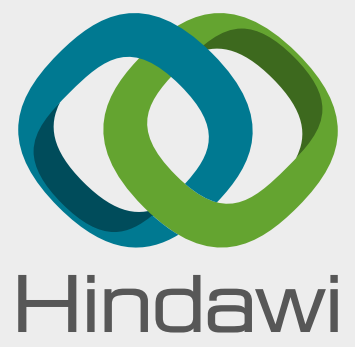

Submit your manuscripts at

www.hindawi.com
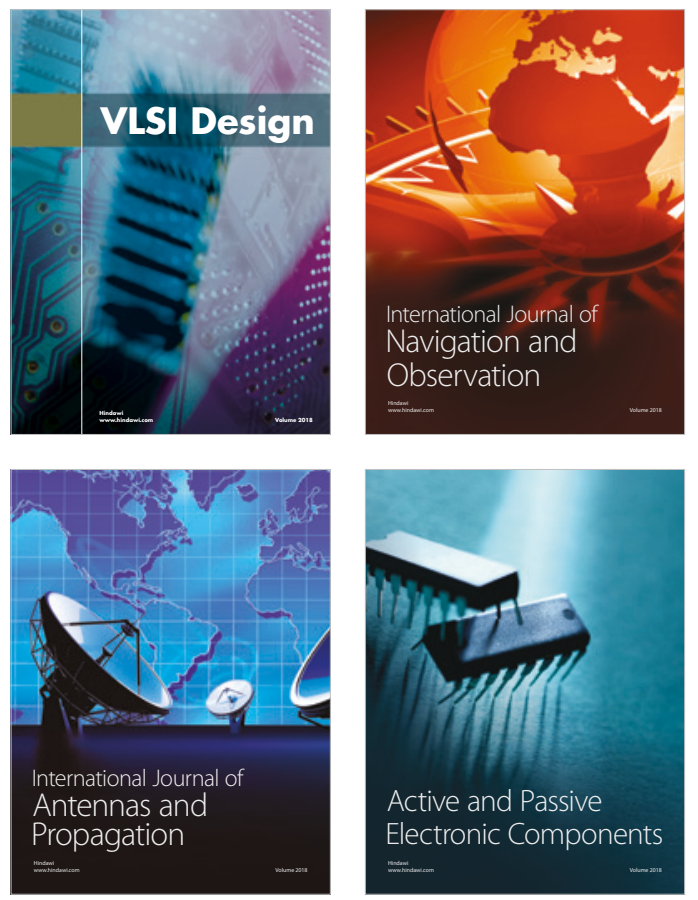
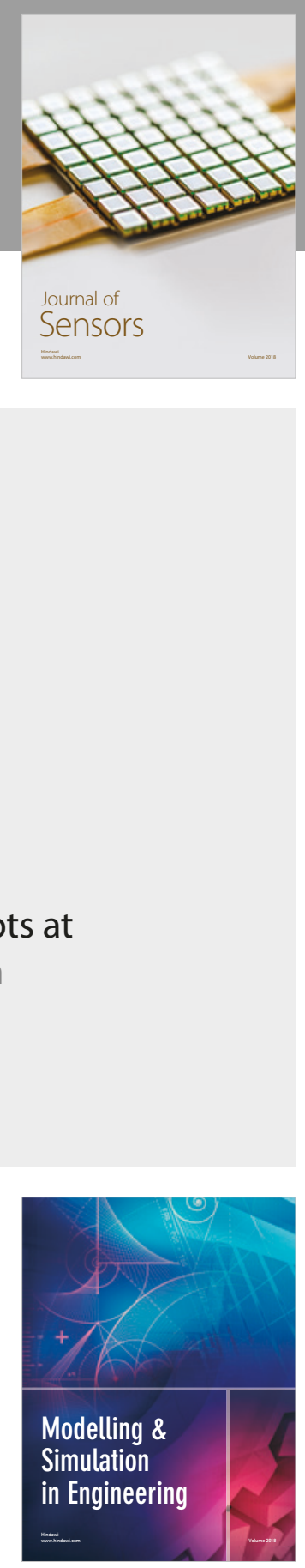

\section{Advances \\ Multimedia}
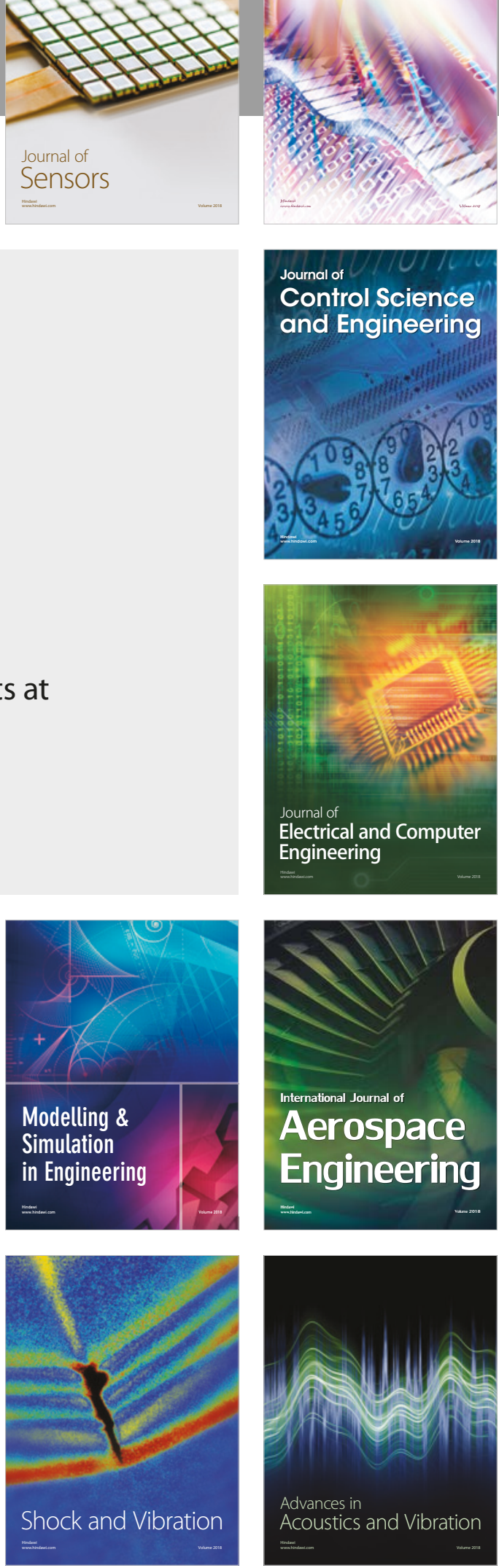